\title{
Simvastatin ameliorates ventricular remodeling via the TGF- $\beta 1$ signaling pathway in rats following myocardial infarction
}

\author{
XIANGBIN XIAO ${ }^{1,2}$, GUANGLEI CHANG ${ }^{1}$, JIAN LIU ${ }^{1}$, GUANGYUN SUN ${ }^{2}$, \\ LI LIU $^{2}$, SHU QIN ${ }^{1}$ and DONGYING ZHANG ${ }^{1}$ \\ ${ }^{1}$ Department of Cardiology, The First Affiliated Hospital of Chongqing Medical University, Chongqing 400016; \\ ${ }^{2}$ Department of Cardiology, The Second People's Hospital of Yibin, Yibin, Sichuan 644000, P.R. China
}

Received May 29, 2015; Accepted March 29, 2016

DOI: $10.3892 / \mathrm{mmr} .2016 .5178$

\begin{abstract}
Statins arewidely used in patients with cardiovascular diseases. A considerable number of previous studies revealed that the intracellular signaling of transforming growth factor (TGF)- $\beta 1$ mediated the development of cardiomyocyte hypertrophy and interstitial fibrosis. However, whether statins can ameliorate ventricular remodeling in post-myocardial infarction via the TGF- $\beta 1$ signaling pathway remains to be rigorously tested. The left anterior descending artery was ligated to induce a rat model of myocardial infarction. The rat model of myocardial infarction was treated with simvastatin through gastric gavage $\left(10,20\right.$ or $\left.40 \mathrm{mg} \mathrm{kg}^{-1} \cdot \mathrm{d}^{-1}\right)$. All rats were sacrificed on day 28 after the myocardial infarction operation. The results revealed that simvastatin significantly improved the hemodynamic indexes, left ventricular mass index, the myocardial tissue structure, the cardiomyocyte cross-sectional area and the collagen volume fraction, and also showed that the levels of TGF- $\beta 1$, TGF-activated kinase (TAK) 1 and drosophila mothers against decapentaplegic (Smad)3 were significantly reduced following treatment with simvastatin, while the levels of Smad7 in the simvastatin treatment groups were markedly increased. The results of the present study suggested that statins ameliorated ventricular remodeling in post-myocardial infarction rats via the TGF- $\beta 1$ signaling pathway, which provided a novel explanation for the pleiotropic effects of statins that benefit the cardiovascular system.
\end{abstract}

\section{Introduction}

Statins, the 3-hydroxy-3-methylglutaryl co-enzyme A reductase inhibitors, are widely used clinically. They exert numerous beneficial effects, particularly in the prevention of cardiovas-

Correspondence to: Professor Dongying Zhang, Department of Cardiology, The First Affiliated Hospital of Chongqing Medical University, 1 Yixueyuan Road, Chongqing 400016, P.R. China E-mail: zdy.chris@gmail.com

Key words: statins, transforming growth factor $\beta 1$ signaling pathway, ventricular remodeling cular diseases. It was previously reported that they can improve endothelial function, alleviate inflammatory reactions, repress ventricular hypertrophy, inhibit the proliferation of fibroblasts and reduce the deposition of interstitial tissue collagen (1-7).

Transforming growth factor (TGF)- $\beta 1$ is now considered to be the quintessential multifunctional cytokine. A considerable number of previous studies showed that TGF- $\beta 1$ intracellular signaling mediated the development of cardiomyocyte hypertrophy and interstitial fibrosis $(8,9)$. TGF-activated kinase (TAK)1 and drosophila mothers against decapentaplegic (Smad) proteins, including Smad1-8, which have been shown to regulate cardiac hypertrophy and remolding, are crucial mediators of the cytostatic effect of TGF- $\beta 1$ (8-11).

Ventricular remodeling in post-myocardial infarction (MI) is a common pathophysiological alteration, as well as an important factor responsible for chronic heart failure (CHF), and $\mathrm{CHF}$ is the leading major cause of mortality in all developed countries. Due to the extraordinary clinical importance of cardiovascular disease and the role of the TGF- $\beta 1$ signaling pathway in cardiac hypertrophy and remodeling, the present study hypothesized that statins mediate their effects via the TGF- $\beta 1$ signaling pathway, however, direct evidence in support of this hypothesis is insufficient. Therefore, the present study aimed to determine whether statins ameliorate ventricular remodeling in post-MI rats via the TGF- $\beta 1$ signaling pathway. For this purpose, simvastatin was widely used in clinical patients used in the present study.

\section{Materials and methods}

Experimental animals and drugs. Male Sprague-Dawley rats (age, 6-8 weeks; weight, 160-240 g) were provided by the Animal Experimental Center of Chongqing Medical University (Sichuan, China) were housed in groups of five on a $12 \mathrm{~h}$ light/dark cycle, with ad libitum access to standard rat chow and water. The rats were subjected to sham-surgery or left coronary artery ligation. The experiments were performed following the approval of the Animal Ethical Committee of the Chongqing Medical University for the use of experiment animals and conform to the Guide for Care and Use of Laboratory Animals. Simvastatin was purchased from Merck Sharp and Dohme (Rahway, NJ, USA). 
Establishment of the MI model. All rats were anesthetized using 3.5\% of $10 \mathrm{ml} / \mathrm{kg}$ chloral hydrate (Qingdao Yulong Algae Co., Ltd., Shandong, China). Tracheotomy was performed for ventilation by a respirator (ALC-V8B; Shanghai Alcott Biotech Co., Ltd., Shanghai, China) with a stroke volume of $28 \mathrm{ml} / \mathrm{kg}$, air pressure of $10 \mathrm{mmHg}$, respiration rate of $1: 1$ and at a rate of 86 strokes/min. The electrocardiogram of lead II was monitored. Thoracotomy was performed and the left anterior descending coronary artery was ligated using 6-0 silk (Shanghai Pudong Jinhuan Medical Products Co., Ltd., Shanghai, China). Fifty rats are randomly divided into five groups ( $\mathrm{n}=10$ in each group), the MI group, the Sim1 group, the Sim 2 group ,the Sim4 group, and the sham-surgery group. All rats underwent MI by left anterior descending coronary artery ligation except for the sham rats, which underwent identical surgery, with the exception of the coronary artery ligation. At $24 \mathrm{~h}$ after MI, one rat had died in the MI group, two rats had died in the Sim1 group and one rat had died in the Sim4 group. At $24 \mathrm{~h}$ after MI the groups were treated as follows: MI ( $=9), 10 \mathrm{mg} / \mathrm{kg} / \mathrm{d}$ simvastatin (Sim1; $\mathrm{n}=8), 20 \mathrm{mg} / \mathrm{kg} / \mathrm{d}$ simvastatin (Sim2; $\mathrm{n}=10) ; 40 \mathrm{mg} / \mathrm{kg} / \mathrm{d}$ simvastatin $(\mathrm{Sim} 4 ; \mathrm{n}=9)$ and sham-surgery animals $(\mathrm{n}=10)$. At $48 \mathrm{~h}$ after the operation, all the Sim-treated groups were administered intragastrically with $4 \%$ simvastatin for 4 consecutive weeks, while the MI and Sham groups were administered intragastrically with the identical quantity of normal saline at 17:30-18:00 on a daily basis.

Hemodynamics measurements. All rats had a recorded body mass (BM; g). The right common carotid artery and left femoral artery were isolated, and a polystyrene PE-20 catheter was inserted into the left ventricle (LV) via the right common carotid artery, with one end connected to the MPA-2000 multichannel physiologic recorder via energy converter to measure heart rate (HR), left ventricular systolic pressure (LVSP), left ventricular end-diastolic pressure (LVEDP) and the rates of maximum positive and negative left ventricular pressure development ( \pm LVdp/dtmax). Additionally, femoral arterial cannulation was performed for simultaneous recording of systolic (SBP) and diastolic arterial pressure (DBP).

Serum lipid analysis. Upon completion, thoracotomy was performed for sampling blood $(2 \mathrm{ml})$ from the left ventricle. This was obtained by centrifugation to determine serum lipids, including total cholesterol (TC), triglyceride (TG), low-density lipoprotein (LDL-C) and high-density lipoprotein (HDL-C). An AU640 automatic biochemistry analyzer and serum-lipid kit (Wako Pure Chemical. Industries Ltd., Osaka, Japan) were used, according to the manufacturer's protocol, for the detection of serum levels of TC, TG, LDL-C and HDL-C.

Histological analysis. The LV mass (LVM; mg), and the ratio of left ventricular mass/body mass (LVMI; $\mathrm{mg} / \mathrm{g}$ ) were measured. Subsequently, the LV was sectioned at a thickness of $3 \mathrm{~mm}$ for paraffin sectioning and the other tissue sections were stored at $-80^{\circ} \mathrm{C}$ for further examinations. The paraffin sections were subjected to hematoxylin and eosin (HE; Beyotime Institute of Biotechnology, Haimen, China) staining. A total of 50 nucleus-centered cardiomyocytes were selected from each specimen and these cardiomyocytes underwent measurement of their cross-sectional area (CSA) using Computer Pathological Image Analytical system (Beijing University of Aeronautics and Astronautics, Beijing, China), and the mean value for each specimen was obtained.

Determination of the collagen volume fraction $(C V F)$. Paraffin sections of rat LV were measured for collagen deposition by Picric-Sirius Red Polarimetry. The slides were placed in $1 \%$ picric-sirius red solution (Sigma-Aldrich, St. Louis, MO, USA) for $45 \mathrm{~min}$. Under polarization microscopy, red or yellow staining is representative of the type I collagen, which is arranged tightly and with strong double refraction, where as green indicated type III collagen, which is porous and thin, with weak double refraction. A total of four visual fields in non-infarction zone (NIZ) were randomly selected on each slide under polarization microscopy (X51-P, Olympus, Tokyo, Japan). The averages of the type I CVF and type III CVF were determined using the Image Pro Plus 6.0 image analysis system (Media Cybernetics, Inc., Rockville, MD, USA) and the collagen I/III ratio was calculated.

Western blot analysis. Protein samples were isolated from the left ventricular myocardium of rats. Left ventricular myocardium lysates were prepared by homogenization in cell lysis buffer (Beyotime Institute of Biotechnology). The protein concentration was determined using a bicinchoninic acid assay (Beyotime Institute of Biotechnology). The protein samples $(40 / 20 \mu \mathrm{g})$ were mixed with $2 \mathrm{X}$ sodium dodecyl sulfate sample loading buffer (Beyotime Institute of Biotechnology) and were subsequently separated on a $12 \%$ polyacrylamide gel and blotted on a nitrocellulose membrane (Beyotime Institute of Biotechnology, Inc). The membranes were blocked with 5\% non-fat milk, followed by incubation (at $4^{\circ} \mathrm{C}$ for $\left.24 \mathrm{~h}\right)$ with antibodies specific for TGF- $\beta 1$ (1:100, sc-146), TAK1 (1:100, sc-7162), Smad3 (1:100, sc-169248), Smad7 (1:100, sc-100140) or $\beta$-actin (1:100; sc-47778) from Santa Cruz Biotechnology, Inc. (Santa Cruz, CA, USA). The membranes were subsequently incubated at $37^{\circ} \mathrm{C}$ for $30 \mathrm{~min}$ with horseradish peroxidase-conjugated goat anti-rabbit (cat. no. ZDR-5306), rabbit anti-goat (cat. no. ZDR-5308) and goat anti-mouse (cat. no. ZDR-5307) immunoglobulin G (1:1,000; all from Zhongshan Goldenbridge Biotechnology Corporation, Beijing, China) and enhanced chemiluminescence detection system (Bio-Rad Laboratories, Hercules, CA, USA) was used for visualization. The grey value was measured using Quantity One software.

Immunohistochemistry. Immunohistochemistry was performed, according to the Streptavidin-Biotin Complex methods (12). Briefly, paraffin sections of rat myocardial tissue were dehydrated and dewaxing, then permeabilized with methanol and heated with a microwave to $100^{\circ} \mathrm{C}$ for $5 \mathrm{~min}$. Following heating, the tissue sections were blocked with goat serum (Beyotime Institute of Biotechnology, Inc.) for $30 \mathrm{~min}$ at room temperature. The samples were then incubated with rabbit anti-Smad7 (1:200; Santa Cruz Biotechnology Inc.) antibody overnight at $4^{\circ} \mathrm{C}$. The sections were subsequently incubated with horseradish peroxidase conjugated-goat anti-rabbit immunoglobulin G (1:150; Zhongshan Goldenbridge Biotechnology Corporation) at room temperature for $30 \mathrm{~min}$. 

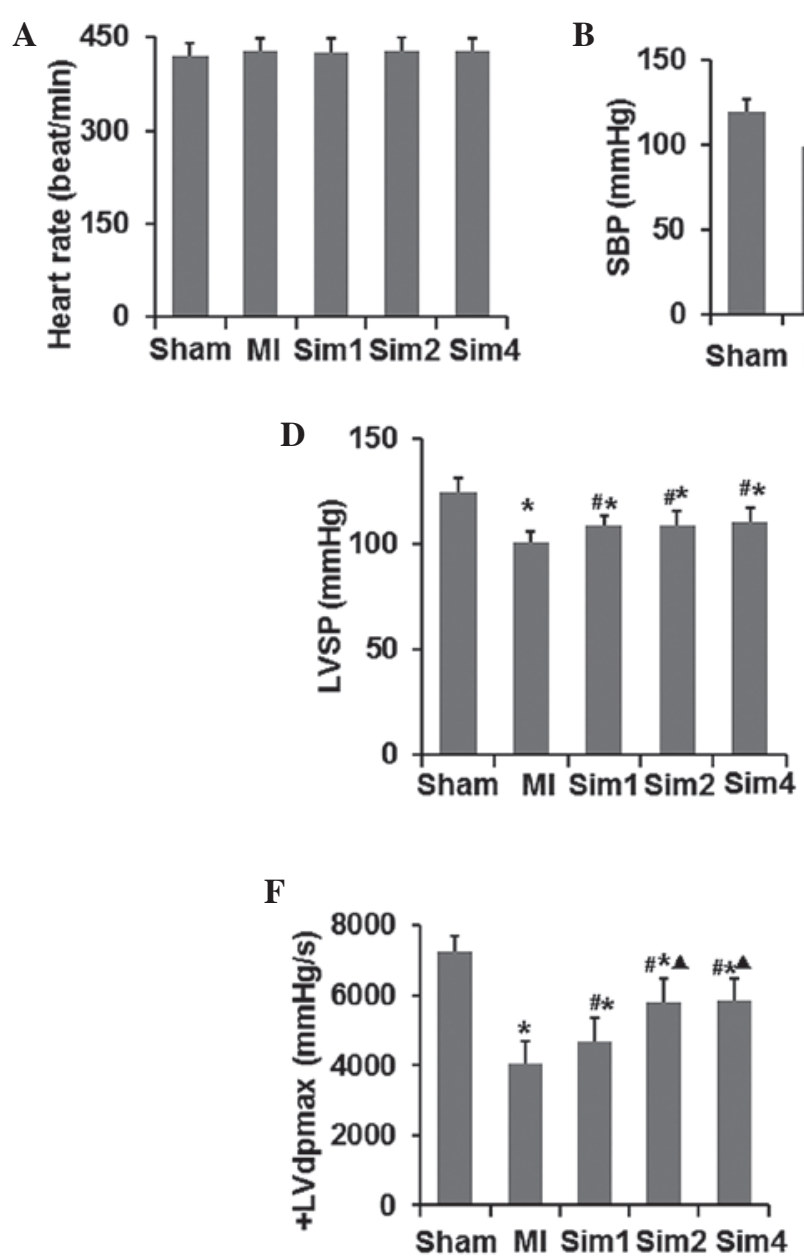
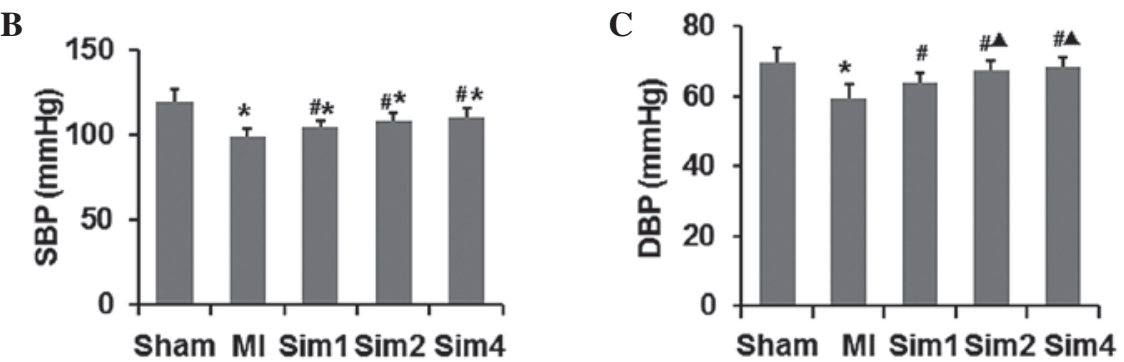

G

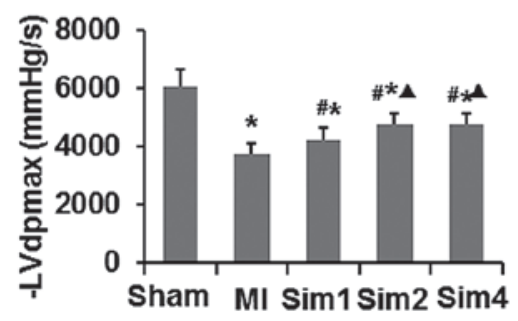

Figure 1. Comparison of hemodynamic parameters in different groups. (A) Heart rate; (B) SBP; (C) DBP; (D) LVSP; (E) LVEDP; (F) +LVdpmax; (G) -LVdpmax. Hemodynamic parameters were determined using an MPA-2000 multichannel physiologic recorder. The data are expressed as the mean \pm standard deviation $\left({ }^{*} \mathrm{P}<0.05\right.$ vs. Sham group; ${ }^{\#} \mathrm{P}<0.05$ vs. MI group; ${ }^{\Delta} \mathrm{P}<0.05$ vs. Sim1 group). MI, myocardial infarction; Sim1, group treated with simvastatin (10 mg/kg/day); Sim2, group treated with simvastatin (20 mg/kg/day); Sim4, group treated with simvastatin (40 mg/kg/day); LVSP, left ventricular systolic pressure; LVEDP, left ventricular end-diastolic pressure; LVdpmax, rate of maximum positive (+) and negative (-) left ventricular pressure development; SBP, systolic arterial pressure; $\mathrm{DBP}$, diastolic arterial pressure.

The samples were then counterstained with HE and washed with phosphate-buffered saline.

Reverse transcription-quantitative polymerase chain reaction (RT-qPCR) analysis. The total RNA was extracted using the Biozol kit, according to the manufacturer's protocol (Beijing Jiamei Niunuo Biotechnology Co., Ltd., Beijing, China). A total of $1 \mu$ l RNA was subjected to RT using an RT-PCR kit (Beijing Jiamei Niunuo Biotechnology Co., Ltd.). The cDNA was PCR-amplified using appropriate primers. Primer sequences used as follows: TGF- $\beta 1$, forward: GACTACGCCAAAGAA GTCAC and reverse: AAGCCACTCAGGCGTATCAG; TAK1, forward: ACAAGTCCCTGCCACAAAC and reverse: GATGGATCTACGCCTTGGTT; Smad3, forward: ATCTAC TG CCG-CTTGTGG and reverse: CTGTGAAGCGTGGAA TGT; Smad7, forward: GGCATTC CTCGGAAGTCA and reverse: AGAAGTTGGGAATCTGAAAGC (Beijing Parkson Gene Technology Co., Ltd., Beijing, China). $\beta$-actin was used as an internal control in RT-qPCR. The PCR products were separated on agarose gels and stained with ethidium bromide. The bands were quantified using Quantity One software 4.62 (Bio-Rad Laboratories) and normalized against $\beta$-actin.
Statistical analysis. SPSS 17.0 software was used for statistical analysis. The data are presented as the mean \pm standard deviation. Differences between the values were determined using Student's t-test. Grouped data were analyzed using a one-way analysis of variance, followed by the Student-Newman-Keuls test. $\mathrm{P}<0.05$ was considered to indicate a statistically significant difference.

\section{Results}

Simvastatin enhances left ventricular function following myocardial injury in MI rats. Firstly, the effects of simvastatin on the hemodynamics were assessed using an MPA-2000 multichannel physiologic recorder, analyzing the HR, LVSP, LVEDP, SBP, DBP and \pm LVdp/dtmax. As shown in Fig. 1, treatment of the rats with simvastatin increased the LVSP, SBP, DBP, \pm LVdp/dtmax and decreased LVEDP; however, these remained lower compared with the rats in the sham group. Additionally, the Sim2 and Sim4 groups revealed an improved LVSP, SBP, DBP \pm LVdp/dtmax and LVEDP compared with the Sim1 gorup. No differences between the Sim 2 and Sim 4 group were observed in any 

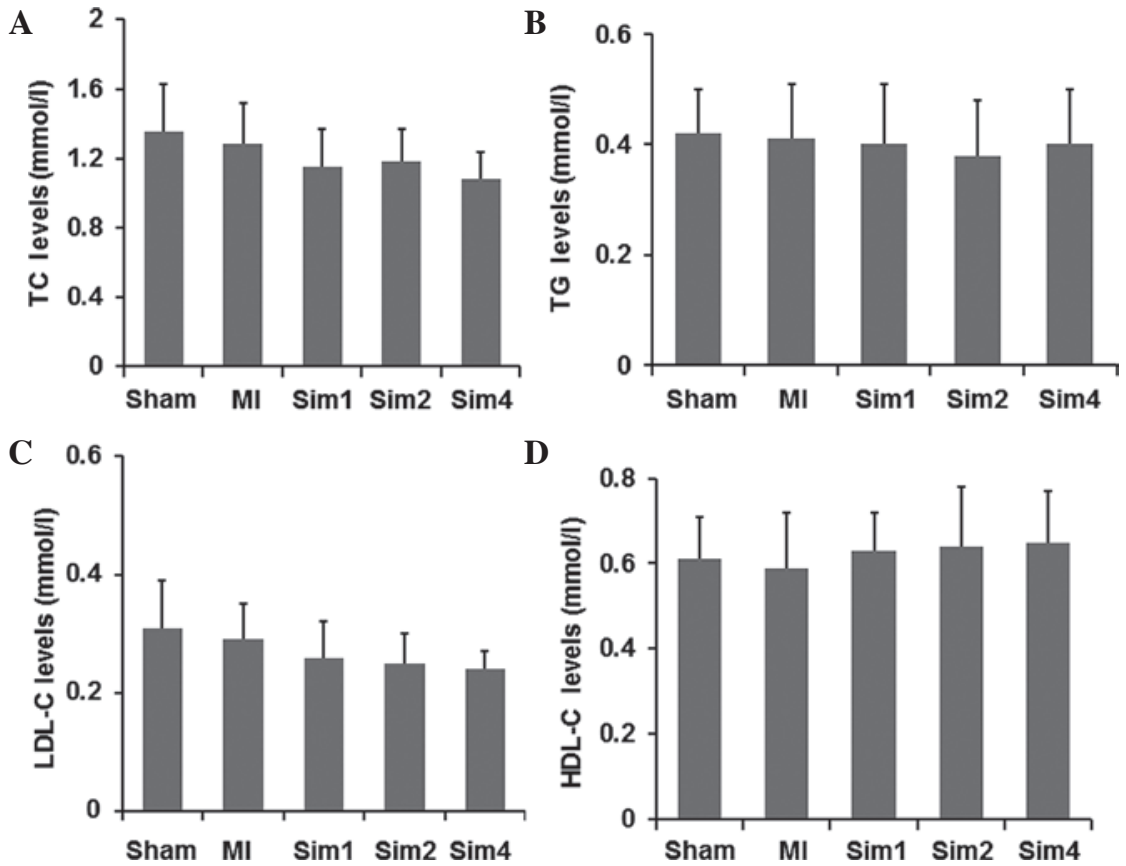

Figure 2. Effects of simvastatin on the serum levels of (A) TC, (B) TG, (C) LDL-C and (D) HDL-C. The data are expressed as the mean \pm standard deviation. MI, myocardial infarction; Sim1, group treated with simvastatin (10 mg/kg/day); Sim2, group treated with simvastatin (20 mg/kg/day); Sim4, group treated with simvastatin (40 mg/kg/day); TC, total cholesterol; TG, triglyceride; LDL-C, low-density lipoprotein; HDL-C, high-density lipoprotein.


Figure 3. Effects of simvastatin on ventricular remodeling. At the end of the experiment, (A) body weight, (B) LVM and (C) LVMI were determined. The data are expressed as the mean \pm standard deviation ( ${ }^{*} \mathrm{P}<0.05$ vs. Sham group; ${ }^{\sharp} \mathrm{P}<0.05$ vs. MI group; ${ }^{\circ} \mathrm{P}<0.05$ vs. Sim 1 group). MI, myocardial infarction; Sim1, group treated with simvastatin (10 mg/kg/day); Sim2, group treated with simvastatin (20 mg/kg/day); Sim4, group treated with simvastatin (40 mg/kg/day); LVM, left ventricle mass; LVMI, the ratio of left ventricular mass/body mass.

hemodynamic parameters. Taken together, these data indicated that left ventricular function was enhanced by simvastatin in MI rats with myocardial injury.

Simvastatin causes no affect on the levels of serum lipids. In order to role out the possible lipid-regulating effects of simvastatin, the basic clinical features of rats were assessed following simvastatin treatment. As shown in Fig. 2, the Sim groups exhibited lower levels of serum lipids compared with the rats in the Sham and MI groups. However, no significant difference was observed in the levels of serum lipid among all groups. These results suggested that the effect of simvastatin in improving post-MI remodeling in rats was independent of its lipid-regulating effect.

Simvastatin ameliorates ventricular remodeling following myocardial injury in MI rats. LVMI is a marker of left ventricular hypertrophy. The LV was sieved and the LVM and LVMI were recorded (Fig. 3). No difference was observed between any of the groups in BM. The LVM and LVMI in the MI and Sim groups were significantly higher compared with the Sham-operated group, while the values were lower in the Sim groups compared with the MI group. The LVM and LVMI in the Sim2 and Sim4 groups were decreased more markedly compared with in the Sim1 group; however, no difference between the Sim2 and Sim4 was detected.

The histopathology and cardiomyocyte CSA $\left(\times 10^{4} \mu \mathrm{m}^{2}\right)$ of the myocardium tissues were also assessed (Fig. 4A). HE staining revealed that the cardiomyocytes in the Sham group were regularly in good condition. The MI group exhibited large infarction size, thin ventricle wall on the infracted region and significantly reduced cardiomyocytes, which were substituted by numerous strip-like fasciculation collagen fibers and were structurally disordered. In the non-infarcted region of MI group, infiltration of considerable inflammatory granulocytes and monocytes, proliferation of protofibrocytes, and compensatory hypertrophy of the survival cardiomyocytes were noted. Compared with the 


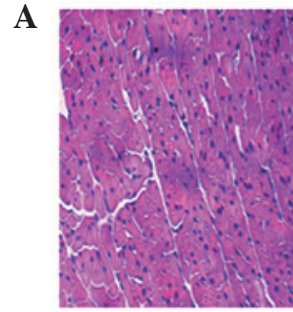

Sham

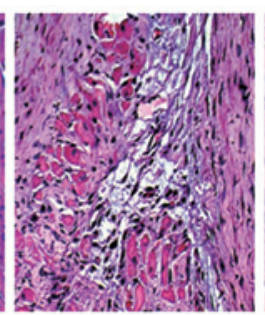

MI

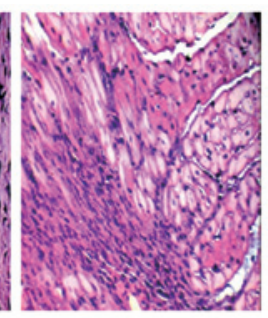

$\operatorname{Sim} 1$

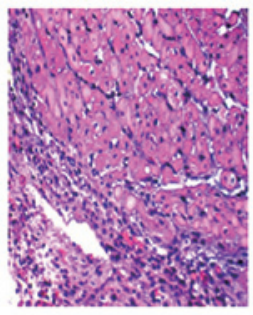

$\operatorname{sim} 2$



$\operatorname{sim} 4$

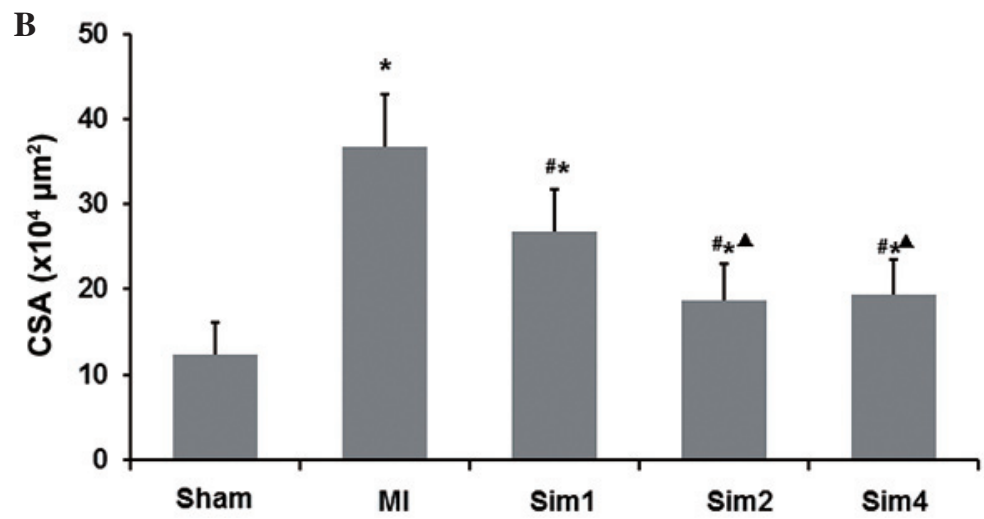

Figure 4. The changes of myocardial tissue structures among groups. (A) Hematoxylin and eosin staining revealed the left ventricles of the myocardium tissues (magnification, $\mathrm{x} 400$ ). (B) The effect of simvastatin on cardiomyocyte CSA was measured by computer pathological image analytical system. The data are expressed as the mean \pm standard deviation ( ${ }^{*} \mathrm{P}<0.05$ vs. Sham-operated group; ${ }^{*} \mathrm{P}<0.05$ vs. MI group; ${ }^{\triangle} \mathrm{P}<0.05$ vs. Sim 1 group). MI, myocardial infarction;

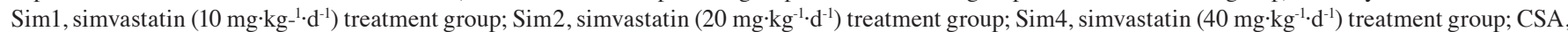
cross-sectional area.

MI group, the Sim groups exhibited significant attenuation of cell degeneration and necrosis along with infiltration of inflammatory cells and less collagen fiber in the infracted region, while in the non-infarcted region, infiltration of numerous inflammatory cells and smaller cardiomyocytes were noted.

Functional assays, shown in Fig. 4B, demonstrated that compared with the MI group, simvastatin reduced the cardiomyocyte CSA $\left(\mathrm{x} 10^{4} \mu \mathrm{m}^{2}\right)$. It was also observed that the CSA was lower in the Sim 2 and Sim4 groups compared with in Sim1 group, while no difference was observed between the Sim2 and Sim 4 groups.

The collagen volume fraction $(\mathrm{CVF})$ was altered in the non-infarcted zone of the LV of all groups. Fig. 5A shows the results of Picric-Sirius Red Polarimetry for type I and type III collagen fiber in the non-infarcted zone of the LV. Quantification of the data (Fig. 5B) revealed that the levels of the type I, type III collagen fiber and I/III were increased significantly in the MI rats compared with those from Sham- and Sim-treatment rats. The data in the Sim2 and Sim 4 groups were also significantly reduced compared with those in the Sim 1 group; however, no differences were observed between the Sim2 and Sim4 groups.

Taken together, these data indicated that simvastatin can ameliorate ventricular remodeling following myocardial injury in MI rats.

Simvastatin inhibits the expression levels of TGF- $\beta 1$, TAK1 and Smad3, and increases the expression of Smad7 following myocardial injury in MI rats. The effects of simvastatin on the mRNA and protein expression levels of TGF- $\beta 1$, TAK1, Smad 3 and Smad7 were examined by RT-qPCR and western blotting (Figs. 6 and 7). As shown in Figs. 6 and 7, the mRNA and protein expression levels of TGF- $\beta 1$, TAK 1 and Smad3 were higher in the non-infarcted zone of the LV in the MI group compared with those from the Sham and Sim groups. While the mRNA and protein expression levels of Smad7 were lower in the MI group compared with those in the Sham and Sim groups. Following treatment with simvastatin, the mRNA and protein expression levels of TGF- $\beta 1$, TAK1 and Smad3 were elevated compared with those in the MI group, while the mRNA and protein expression levels of Smad7 in the Sim groups were significantly decreased compared with those in the MI group. Notably, both the mRNA and protein expression levels revealed no difference between the Sim 2 and Sim 4 groups.

Therefore, the present study speculated that simvastatin ameliorated ventricular remodeling via the TGF- $\beta 1$ signaling pathway in post-MI rats.

\section{Discussion}

The present study investigated the protective effects of simvastatin treatment on ventricular remodeling in the MI rat model. Following pre-treatment with simvastatin in MI rats, it was revealed that the left ventricular function was enhanced. Additionally, the ventricular remodeling markers, including LVMI, CSA and CVF were inhibited. These data indicated that simvastatin meliorated ventricular remodeling in the MI rat model. RT-qPCR and western blotting also showed that the expression levels of TGF- $\beta 1$, TAK1 and Smad3 were reduced, and the expression of Smad7 was increased. Therefore, it was 


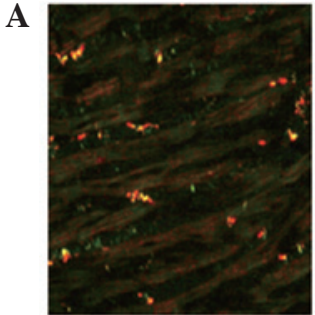

Sham

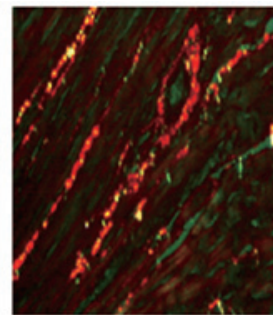

MI



$\operatorname{Sim} 1$



$\operatorname{Sim} 2$

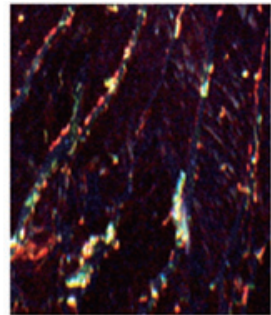

$\operatorname{sim} 4$

B

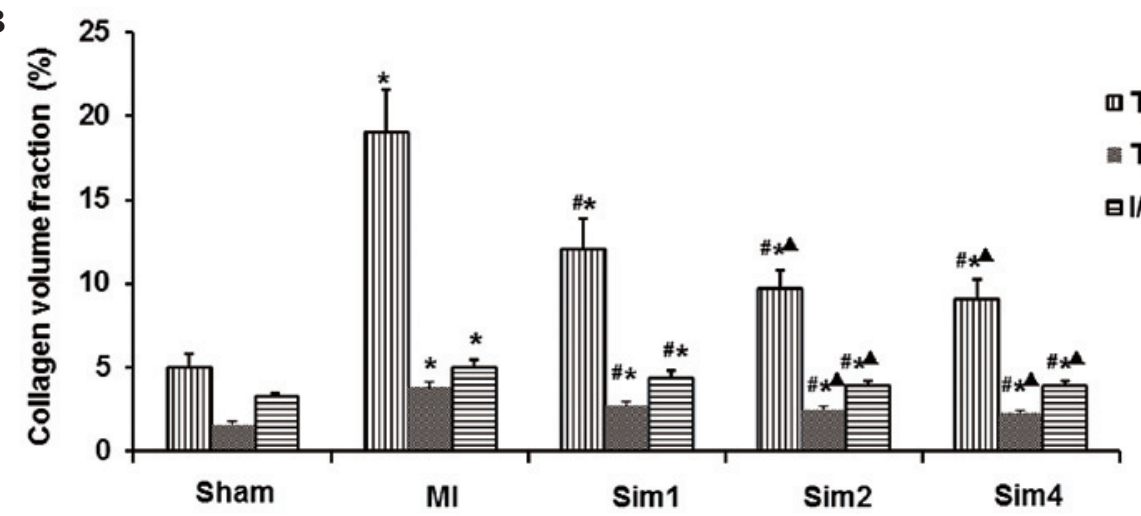

Figure 5. Simvastatin inhibited collagen deposition in myocardium tissues from the rats in all groups. (A) The left ventricle myocardium tissue was stained with Picric-Sirius Red to evaluate tissue fibrosis (magnification, x100). Type I collagen (red/yellow) and type III collagen (green) were visualized. (B) The effect of simvastatin on type I, type III and I/III collagen were determined using the Image Pro Plus 6.0 image analysis system. MI, myocardial infarction; Sim1, simvastatin $\left(10 \mathrm{mg} \cdot \mathrm{kg}^{-1} \cdot \mathrm{d}^{-1}\right)$ treatment group; Sim 2 , simvastatin $\left(20 \mathrm{mg} \cdot \mathrm{kg}^{-1} \cdot \mathrm{d}^{-1}\right)$ treatment group; Sim4, simvastatin $\left(40 \mathrm{mg} \cdot \mathrm{kg}^{-1} \cdot \mathrm{d}^{-1}\right)$ treatment group. The data are expressed as the mean \pm standard deviation ( $\left(\mathrm{P}<0.05\right.$ vs. Sham-operated group; ${ }^{\mathrm{P}} \mathrm{P}<0.05$ vs. MI group; ${ }^{\wedge} \mathrm{P}<0.05$ vs. Sim1 group).
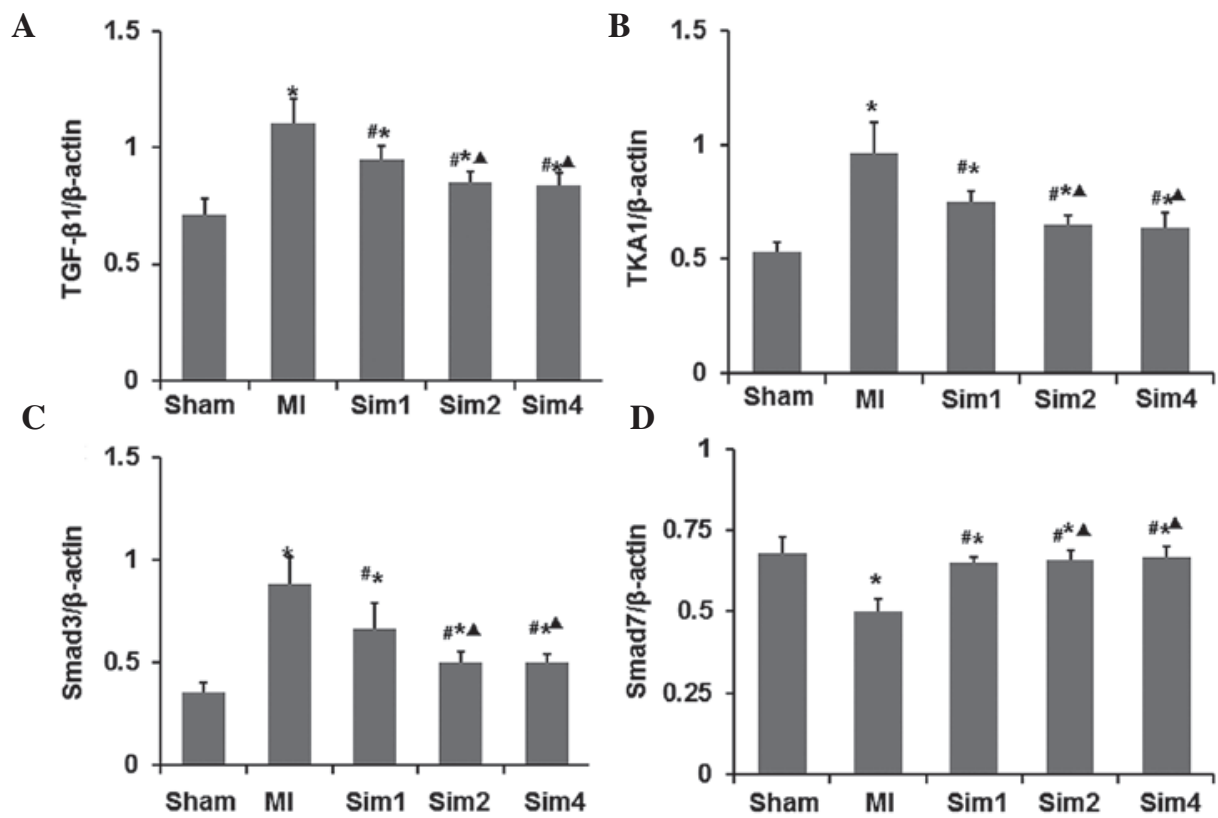

Figure 6. Inhibitory effects of simvastatin on the mRNA expression levels of TGF- $\beta 1$, TAK1 and Smad 3 in the left ventricles. The mRNA expression levels of (A) TGF- $\beta 1$, (B) TAK1, (C) Smad3 and (D) Smad7 were determined by RT-qPCR and the data are expressed as the ratio against $\beta$-actin. The data are expressed as the mean \pm standard deviation ( ${ }^{*} \mathrm{P}<0.05$ vs. Sham-operated group; ${ }^{*} \mathrm{P}<0.05$ vs. MI group; ${ }^{\wedge} \mathrm{P}<0.05$ vs. Sim1 group). MI, myocardial infarction; Sim1: simvastatin $\left(10 \mathrm{mg} \mathrm{kg}^{-1} \cdot \mathrm{d}^{-1}\right)$ treatment group; Sim 2 , simvastatin $\left(20 \mathrm{mg} \cdot \mathrm{kg}^{-1} \cdot \mathrm{d}^{-1}\right)$ treatment group; Sim 4 , simvastatin $\left(40 \mathrm{mg} \cdot \mathrm{kg}^{-} \cdot{ }^{1} \cdot \mathrm{d}^{-1}\right)$ treatment group; TGF, transforming growth factor; TAK, TGF-activated kinase; Smad, drosophila mothers against decapentaplegic protein; RT-qPCR, reverse transcription-quantitative polymerase chain reaction.

speculated that simvastatin reduced ventricular remodeling in MI rats via TGF- $\beta 1$, TAK1 and Smad3 decrease, and Smad7 increase.
Several lines of evidence have demonstrated that the TGF- $\beta 1$ signaling pathway is key role in the pathogenesis of ventricular remodeling following MI (8-11). In the present study, 
A

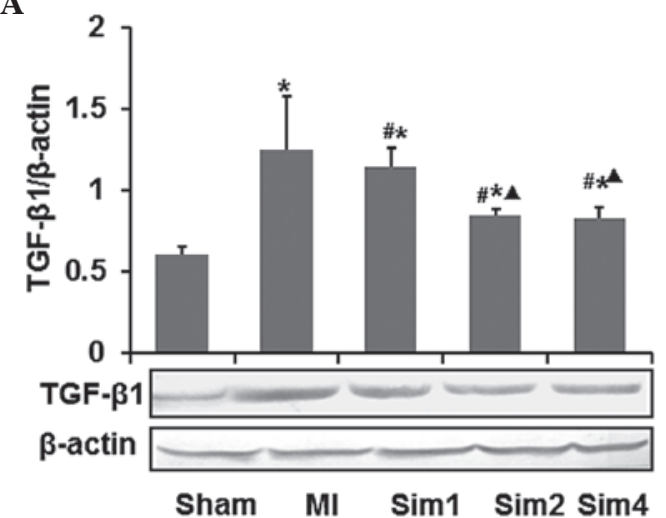

C

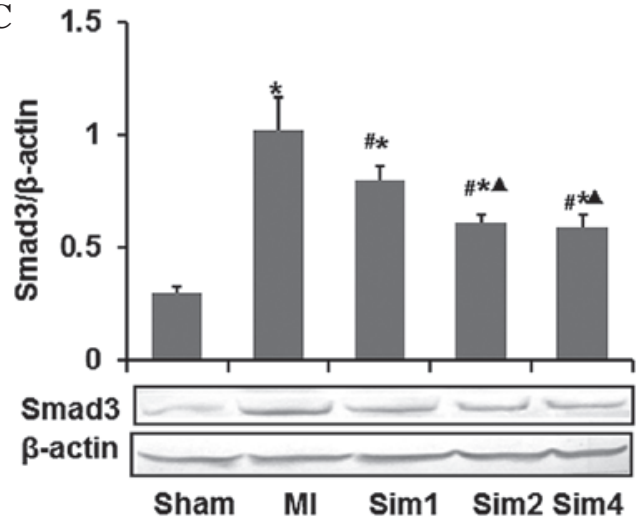

B

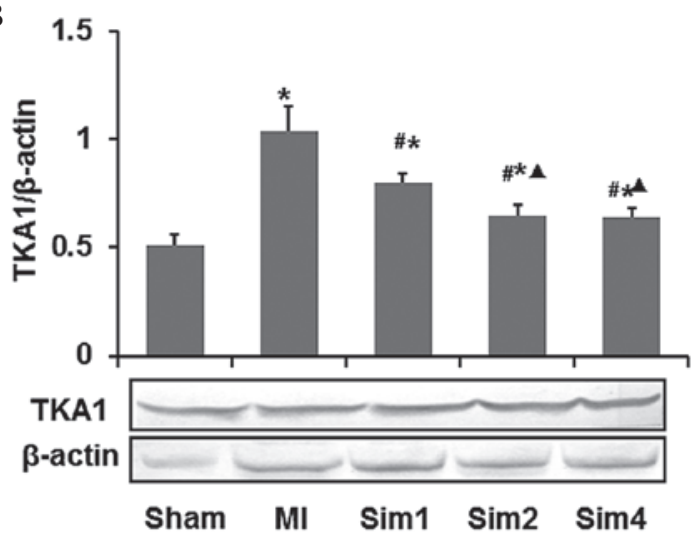

D

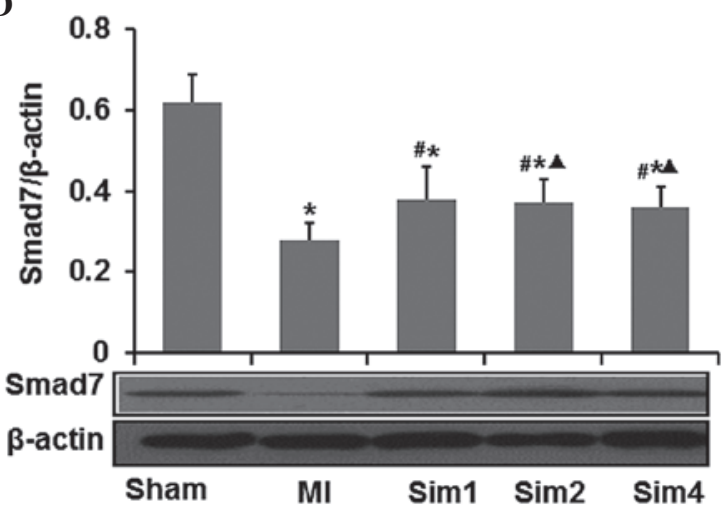

Figure 7. Inhibitory effects of simvastatin on the protein expression levels of TGF- $\beta 1$, TAK1 and Smad 3 in the left ventricles. The levels of (A) TGF- $\beta 1$, (B) TAK1, (C) Smad3 and (D) Smad7 were determined by western blotting and the data are expressed as the ratio against $\beta$-actin. The data are expressed as the mean \pm standard deviation $\left({ }^{*} \mathrm{P}<0.05\right.$ vs. Sham-operated group; ${ }^{*} \mathrm{P}<0.05$ vs. MI group; ${ }^{\Delta} \mathrm{P}<0.05$ vs. Sim 1 group). MI, myocardial infarction; Sim1: simvastatin $\left(10 \mathrm{mg} \mathrm{kg}^{-1} \cdot \mathrm{d}^{-1}\right)$ treatment group; Sim 2 , simvastatin $\left(20 \mathrm{mg} \cdot \mathrm{kg}_{-}{ }^{1} \cdot \mathrm{d}^{-1}\right)$ treatment group; Sim4, simvastatin $\left(40 \mathrm{mg} \cdot \mathrm{kg}{ }^{1} \cdot \mathrm{d}^{-1}\right)$ treatment group; TGF, transforming growth factor; TAK, TGF-activated kinase; Smad, drosophila mothers against decapentaplegic protein.

simvastatin was used to demonstrate the positive effects of statins on the ventricular remodeling following MI and provided a novel role of TGF- $\beta 1$ signaling in the positive effect of statins on ventricular remodeling.

In the present study, the effects of simvastatin on the hemodynamics were assessed and revealed that the LVSP and \pm LVdp/dtmax were decreased, while LVEDP increased significantly following MI. This indicated that both systolic and diastolic functions in MI rats were seriously damaged. However, these pathological changes were improved in the simvastatin treated rats. Structurally, in the MI rats, necrosis and apoptosis of cardiomyocytes was observed, ventricular remodeling was confirmed by increased LVMI and interstitial collagen deposition, and it was directly correlated to functional alteration in respective groups mentioned above. Furthermore, treatment with simvastatin ameliorated all these changes.

LVMI, a marker of left ventricular hypertrophy, was increased in the MI and simvastatin-treated groups compared with the Sham-operated group. These occurrences signified the presence of post-MI remodeling, evidenced by increased LVMI, and interstitial collagen deposition was decreased by the treatment of simvastatin for 4 weeks compared with the MI group. Guo et al (13) also demonstrated decreased LVMI by treatment of simvatatin for 4 weeks in post-MI rats, which is consistent with the present findings, demonstrating the effect of simvastatin in improving post-MI ventricular remodeling.
The present study also demonstrated increased CSA in the non-infarcted region following $\mathrm{MI}$; however, this was decreased by simvastatin treatment. This finding is consistent with other previous studies $(4,14)$, suggesting the effect of simvastatin in improving post-MI ventricular function, and this effect may be attributed to its activities in inhibiting ventricular hypertrophy post-MI and improving the compliance of ventricular wall. The deposition of myocardial interstitial collagen and the disproportion of collagen type I/III are partly responsible for decreased compliance of ventricular wall and impairment of heart function. The present data suggested that in the post-MI group compared with the Sham group, the type I and type III CVFs were significantly elevated, with the collagen type I/III ratio altered in NIZ. This resulted in increased proliferation, synthesis and secretion of myocardial fibroblasts, which resulted in the disproportion of collagen types and remodeling of myocardial collagen lattice spatial structure. Compared with the MI group, type I and type III CVFs in the NIZ were significantly decreased, accompanied by normalization of collagen ratio, after 4 weeks treatment with simvastatin. This suggested the effect of simvastatin in decreasing collagen content, ameliorating the ratio of collagen types, improving interstitial remodeling and increasing compliance of ventricular wall, in accordance to that reported previously (15-17). Serum levels of TG, TC, HDL-C and LDL-C in the post-MI rats after 4 weeks remained significantly unaltered in all simvastatin-treated groups as compared with those in the MI or 
Sham-operated groups. This suggested that the treatment with simvastatin for 4 weeks had little impact on serum lipids in post-MI rats, and this finding is consistent with those reported overseas $(18,19)$, confirming that the effect of simvastatin in improving post-MI remodeling in rats is independent of its lipid-regulating effect.

TGF- $\beta$ is a type of multifunctional cytokine, including three highly homologous TGF- $\beta$ isoforms (TGF- $\beta 1,2$ and 3 ). TGF- $\beta 1$ is the prevalent isoform and is found almost ubiquitously, whereas the other isoforms are expressed in a more limited spectrum of cells and tissues (20). TGF- $\beta 1$ signaling has a wide variety of biological and pathological actions, and it is one of the most important factors responsible for cardiac remodeling in cases of myocardial damage or cardiac overload (5).

TAK1, a member of the mitogen-activated protein kinase kinase kinase and the downstream substrate of TGF- $\beta 1$ signal transduction, has a close correlation with cardiomyocyte hypertrophy and cardiac failure $(6,10)$. The present study positively demonstrated a significantly increased expression of TGF- $\beta 1$ and TAK1 at the transcriptional and translational level in the non-infarcted region in post-MI rats as compared with the Sham-operated rats. However, it was significantly downregulated by the simvastatin treatment. Previous studies elsewhere found that the TGF- $\beta 1$ and TAK1 expression in the non-infarcted region following MI increased significantly as compared with the control, and subsequent immunohistochemical staining revealed that TAK1 was chiefly located in cardiomyocytes. This implied that the activation of the TGF- $\beta 1 /$ TAK1 signaling pathway in the non-infarcted region accounted for cardiomyocyte hypertrophy following MI (21). The present study suggested that pleiotropic effects of simvastatin may be attributed to its role in downregulating the expression of TGF- $\beta 1 /$ TAK 1 and inhibiting intracellular signaling of the TGF- $\beta 1 /$ TAK1 pathway, and by doing so, improved post MI-associated ventricular remodeling.

TGF- $\beta 1 /$ Smads signal transduction is induced and rapidly activated in cases of cardiac cell damage or infarct healing, and may be important in modulating inflammatory reaction and fibrosis $(5,11)$. With regards to the association between the activation of the TGF- $\beta 1 / \mathrm{Smad} 3$ signaling pathway myocardial interstitial remodeling, the present study demonstrated low level expression of TGF- $\beta 1$ and Smad 3 in normal myocardium and significantly increased the expression of TGF- $\beta 1$ and Smad3 in the non-infarcted region in post-MI rats. This was accompanied with increased collagen content and collagen type I/III ratio alteration. It was previously demonstrated that gene knockdown regulating Smad3 expression prevents interstitial fibrosis in the non-infarcted myocardium and attenuates further cardiac remodeling (6). Accordingly, SIS3, a reagent with selective inhibition of Smad3, can inhibit excessive extracellular matrix production by inhibiting TGF- $\beta 1$ signaling (22). The expression of TGF- $\beta 1$ and Smad 3 in the non-infarcted region was remarkably decreased by simvastatin for 4 weeks, accompanied by decreased collagen content and the amelioration of collagen type I/III ratio. This expression altogether augmented hemodynamic parameters, suggesting that simvastatin improves post-MI ventricular remodeling via its effects in downregulating the expression of TGF- $\beta 1 / \mathrm{Smad} 3$ and inhibiting intracellular signaling of the TGF- $\beta 1 / \mathrm{Smad} 3$ pathway.
Smad7 is different from Smad3, in that it is an inhibitory type subunit in TGF- $\beta 1 / \mathrm{Smads}$ signal transduction and is negatively regulated by this pathway (9). The present study demonstrated that the alteration of Smad7 is conversed with TGF- $\beta 1$ and Smad 3 in all groups, and increased expression of Smad7 accompanied by the amelioration of ventricular remodeling, suggesting that simvastatin improves post-MI ventricular remodeling attributed to its effects in upregulating the expression of Smad7.

It had been proved medically that early administration of large doses of statins as an intensive therapy for patients with acute coronary syndrome can lower the incidence of coronary artery events (23-27). In the present study, three groups with different simvastatin dosages were set up to observe their effects on ventricular remodeling. The present study demonstrated that Sim 2 and Sim4 were superior compared with Sim1 in efficacy by improving ventricular remodeling and hemodynamic parameters, with comparable Sim2 and Sim4 potency, suggesting that for MI model rats, simvastatin exhibited a dose-dependent manner with $20 \mathrm{mg} \cdot \mathrm{kg}^{-1} \cdot \mathrm{d}^{-1}$ as potential therapeutic range, however, not exceeding this dose.

In conclusion, simvastatin treatment may significantly improve ventricular remodeling following MI, inhibit cardiomyocyte hypertrophy and interstitial fibrosis, improve the compliance of ventricular wall and improve cardiac function. These outcomes proved to be independent of its lipid-regulating effect, but achieved through intracellular signal transduction of TGF- $\beta 1$ inhibition.

\section{Acknowledgements}

The present study was supported by the National Natural Science Fund (no. 81570212), the Natural Science Foundation Project of CQ CSTC (no. cstc2011jjA10008), the Chongqing Municipal Health Bureau fund (nos. 2010-1-07, 2012-2-125 and ZY20132124), and the National key Clinical Specialties Construction Program of China (no. 2011-170). The authros would like to thank Mr. Jianyong Wu and Mr. Dezhang Zhao (Institute of Life Sciences, Chongqing Medical University, Chongqing, China) for their excellent technical support for the flow cytometry analysis.

\section{References}

1. Wassmann S, Laufs U, Bäumer AT, Müller K, Ahlbory K, Linz W, Itter G, Rösen R, Böhm M and Nickenig G: HMG-CoA reductase inhibitors improve endothelial dysfunction in normocholesterolemic hypertension via reduced production of reactive oxygen species. Hypertension 37: 1450-1457, 2001.

2. Stumpf C, Petzi S, Seybold K, Wasmeier G, Arnold M, Raaz D, Yilmaz A, Daniel WG and Garlichs CD: Atorvastatin enhances interleukin-10 levels and improves cardiac function in rats after acute myocardial infarction. Clin Sci (Lond) 116: 45-52, 2009.

3. Porter KE and Turner NA:Statins and myocardial remodelling: Cell and molecular pathways. Expert Rev Mol Med 13: e22, 2011.

4. Correale M, Abruzzese S, Greco CA, Concilio M, Biase MD and Brunetti ND: Pleiotropic effects of statin in therapy in heart failure: A review. Curr Vasc Pharmacol 12: 873-884, 2014.

5. Bonifacio A, Mullen PJ, Mityko IS, Navegantes LC, Bouitbir J and Krähenbühl S: Simvastatin induces mitochondrial dysfunction and increased atrogin-1 expression in H9c2 cardiomyocytes and mice in vivo. Arch Toxicol, 2014 (Epub ahead of print). 
6. Node K, Fujita M, Kitakaze M, Hori M and Liao JK: Short-term statin therapy improves cardiac function and symptoms in patients with idiopathic dilated cardiomyopathy. Circulation 108 839-843, 2003

7. Horwich TB, MacLellan WR and Fonarow GC: Statin therapy is associated with improved survival in ischemic and non-ischemic heart failure. J Am Coll Cardio 43: 642-648, 2004.

8. Li L, Chen Y, Doan J, Murray J, Molkentin JD and Liu Q: Transforming growth factor $\beta$-activated kinase 1 signaling pathway critically regulates myocardial survival and remodeling. Circulation 130: 2162-2172, 2014.

9. Wang BW, Wu GJ, Cheng WP and Shyu KG: Mechanical stretch via transforming growth factor- $\beta 1$ activates microRNA-208a to regulate hypertrophy in cultured rat cardiac myocytes. J Formos Med Assoc 112: 635-643, 2013.

10. Shi Y and Massagué J: Mechanisms of TGF-beta signaling from cell membrane to the nucleus. Cell 113: 685-700, 2003.

11. Ding YF, Peng YR, Li J, Shen H, Shen MQ and Fang TH: Gualou xiebai decoction prevents myocardial fibrosis by blocking TGF-beta/Smad signalling. J Pharm Pharmacol 65: 1373-1381, 2013.

12. Zhang HY, Chu JF, Li P, Li N and Lv ZH: Expression and diagnosis of transient receptor potential vanilloid1 in urothelium of patients with overactive bladder. J Biol Regul Homeost Agents 29: 875-879, 2015.

13. Guo Y, Shi DZ, Yin HJ and Chen KJ: Effects of tribuli saponins on ventricular remodeling after myocardial infarction in hyperlipidemic rats. Am J Chin Med 35: 309-316, 2007.

14. Nishikawa H, Miura S, Zhang B, Shimomura H, Arai H, Tsuchiya Y, Matsuo K and Saku K: Statins induce the regression of left ventricular mass in patients with angina. Circ J 68: 12l-125, 2004

15. Li TS, Takahashi M, Suzuki R, Kobayashi T, Ito H, Mikamo A and Hamano K: Pravastatin improves remodeling and cardiac function after myocardial infarction by an antiinflammatory mechanism rather than by the induction of angiogenesis. Ann Thorac Surg 81: 2217-2225, 2006.

16. Martin J, Denver R, Bailey M and Krum H: In vitro inhibitory effects of atorvastatin on cardiac fibroblasts: Implications for ventricular remodeling. Clin Exp Pharmacol Physiol 32: 697-701, 2005.

17. Zhang DY, Qin S, Tang XJ and Yang H: Effect of simvastatin on left ventricular remodeling and heart function in rats with myocardial infarction. Chin Pharmacol Bull 22: 814-818, 2006.
18. Bauersachs J, Galuppo P, Fraccarollo D, Christ M and Ertl G: Improvement of left ventricular remodeling and function by hydroxymethylglutaryl coenzyme a reductase inhibition with cerivastatin in rats with heart failure after myocardial infarction. Circulation 104: 982-985, 2001.

19. Zhang J, Cheng X, Liao YH, Lu B, Yang Y, Li B, Ge H, Wang M, Liu Y, Guo Z and Zhang L: Simvastatin regulates myocardial cytokine expression and improves ventricular remodeling in rats after acute myocardial infarction. Cardiovasc Drugs Ther 19: 13-21, 2005

20. Flanders KC: $\operatorname{Smad} 3$ as a mediator of the fibrotic response. Int J Exp Pathol 85: 47-64, 2004.

21. Matsumoto-Ida M, Takimoto Y, Aoyama T, Akao M, Takeda T and Kita T: Activation of TGF-beta1-TAK1-p38 MAPK pathway in spared cardiomyocytes is involved in left ventricular remodeling after myocardial infarction in rats. Am J Physiol Heart Circ Physiol 290: H709-H715, 2006.

22. Jinnin M, Ihn H and Tamaki K: Characterization of SIS3, a novel specific inhibitor of smad 3 and its effect on transforming growth factor-betal-induced extracellular matrix expression. Mol Pharmacol 69: 597-607, 2006.

23. Johnson C, Waters DD, DeMicco DA, Breazna A, Bittner V, Greten H, Grundy SM and LaRosa JC: Comparison of effectiveness of atorvastatin $10 \mathrm{mg}$ versus $80 \mathrm{mg}$ in reducing major cardiovascular events and repeat revascularization in patients with previous percutaneous coronary intervention (post hoc analysis of the Treating to New Targets [TNT] Study). Am J Cardiol 102: 1312-1317, 2008

24. Reddy G and Bittner V: LDL lowering after acute coronary syndrome: Is lower better? Curr Treat Options Cardiovasc Med 15: 33-40, 2013.

25. Colivicchi F, Tubaro M and Santini M: Clinical implications of switching from intensive to moderate statin therapy after acute coronary syndromes. Int J Cardiol 152: 56-60, 2011.

26. Shepherd J, Kastelein JJ, Bittner V, Deedwania P, Breazna A, Dobson S, Wilson DJ, Zuckerman A and Wenger NK; TNT (Treating to New Targets) Investigators. Intensive lipid lowering with atorvastatin in patients with coronary heart disease and chronic kidney disease: The TNT (Treating to New Targets) study. J Am Coll Cardiol 51: 1448-1454, 2008.

27. Apiyasawat S, Sritara P, Ngarmukos T, Sriratanasathavorn C and Kasemsuwan P: Association of statin therapy with ventricular arrhythmias among patients with acute coronary syndrome. Heart Asia 5: 39-41, 2013. 